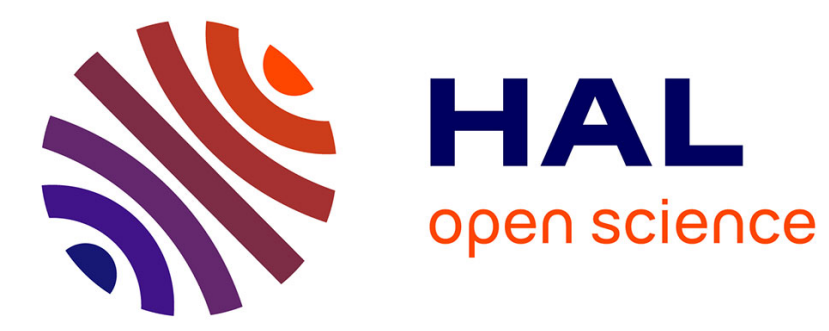

\title{
Les supraconducteurs à base de $\mathrm{Nb}$
}

O. Bethoux

\section{To cite this version:}

O. Bethoux. Les supraconducteurs à base de Nb. Revue de Physique Appliquée, 1970, 5 (3), pp.495503. 10.1051/rphysap:0197000503049500 . jpa-00243420

\section{HAL Id: jpa-00243420 https://hal.science/jpa-00243420}

Submitted on 1 Jan 1970

HAL is a multi-disciplinary open access archive for the deposit and dissemination of scientific research documents, whether they are published or not. The documents may come from teaching and research institutions in France or abroad, or from public or private research centers.
L'archive ouverte pluridisciplinaire HAL, est destinée au dépôt et à la diffusion de documents scientifiques de niveau recherche, publiés ou non, émanant des établissements d'enseignement et de recherche français ou étrangers, des laboratoires publics ou privés. 


\title{
LES SUPRACONDUCTEURS A BASE DE Nb
}

\author{
O. BETHOUX \\ Centre de Recherches sur les Très Basses Températures, 38, Grenoble
}

\begin{abstract}
Résumé. - Après avoir rappelé comment la microstructure d'un matériau influence ses propriétés supraconductrices les plus importantes, on examine le comportement supraconducteur des alliages ductiles et des composés intermétalliques de $\mathrm{Nb}$ en fonction des traitements métallurgiques qui en affectent la microstructure. On expose enfin les causes principales de l'instabilité des supraconducteurs à fortes densités de courant critique et les palliatifs technologiques qui lui ont été apportés sur les matériaux commerciaux.
\end{abstract}

\begin{abstract}
After a short recapitulation of the effect of microstructure on the most important properties of superconductors, the superconducting behaviour of ductiles $\mathrm{Nb}$ alloys and intermetallic $\mathrm{Nb}$ compounds, is examined as a function of metallurgical treatment. Causes of instability in high critical current superconductors and their technological solutions are discussed.
\end{abstract}

Introduction. - Dès les premières recherches, il y a une dizaine d'années, sur les supraconducteurs en hauts champs magnétiques, les matériaux à base de niobium se sont révélés comme les plus prometteurs en vue des applications pour la production des champs magnétiques intenses. A l'heure actuelle ce sont les seuls matériaux supraconducteurs à faire l'objet d'une fabrication industrielle. Des conducteurs destinés à la confection de bobinage sont produits sous forme de fils, câbles, rubans et sont, en fait, composites puisque constitués d'une âme en matériau supraconducteur enrobée dans un métal bon conducteur, $\mathrm{Cu}$ ou $\mathrm{Al}$, destiné à stabiliser le matériau supraconducteur comme nous le verrons plus loin.

Les matériaux actuellement utilisés, essentiellement les alliages $\mathrm{Nb}$-Ti et le composé $\mathrm{Nb}_{3}-\mathrm{Sn}$ appartiennent à la vaste classe des supraconducteurs de $2^{\mathrm{e}}$ espèce. Ils sont capables de transporter de très fortes densités de courant continu $\left(10^{4}\right.$ à $\left.10^{5} \mathrm{~A} . \mathrm{cm}^{-2}\right)$ dans des champs magnétiques supérieurs à 100 kOe. Néanmoins leurs performances dépendent très fortement de leur microstructure et donc des traitements métallurgiques qu'ils ont subi au cours de leur fabrication.

Encore très imparfaitement connues, les relations étroites entre la microstructure et les propriétés supraconductrices des matériaux, constituent un important domaine d'intérêt pour le métallurgiste et c'est sur elles que l'accent sera plus particulièrement mis ici.

Dans une première partie, nous rappellerons rapidement quelles sont les caractéristiques importantes d'un matériau supraconducteur et de quelle manière elles sont influencées, en général, par la composition et la microstructure du matériau.

Dans une deuxième partie nous examinerons plus particulièrement le comportement des alliages et composés intermétalliques de $\mathrm{Nb}$; dans une courte troisième partie nous aborderons la question de la stabilisation des matériaux supraconducteurs.

Première partie. - LeS CARACTÉRISTIQUES SUPRACONDUCTRICES D'UN MATÉRIAU ET LES FACTEURS DONT ELLES DÉPENDENT. - La résistivité électrique de certains matériaux devient brutalement nulle au-dessous d'une certaine température critique, $T_{\mathrm{c}}$ : le matériau est alors supraconducteur. Pour le ramener à l'état normal on peut soit élever sa température au-dessus de $T_{\mathrm{c}}$, soit le soumettre à un champ magnétique supérieur à un certain champ critique $H_{\mathrm{u}}$, soit le faire parcourir par un courant électrique tel que la densité de courant dans le matériau soit supérieure à une certaine valeur $J_{c}$. $J_{c}$ décroît lorsque le champ magnétique dans lequel est placé le matériau croît, pour s'annuler à $H_{\mathrm{u}} ; H_{\mathrm{u}}$ et $J_{\mathrm{c}}$ décroissent lorsque la température augmente pour s'annuler à $T_{\mathrm{c}}$.

Les supraconducteurs étant toujours utilisés pour des raisons de commodités à $4,2 \circ \mathrm{K}$, température d'ébullition de l'hélium sous pression atmosphérique, les valeurs des champs critiques $H_{\mathrm{u}}$ et des densités de courant critique $J_{c}$ dont il sera question par la suite, seront toujours, sauf spécifications contraires, celles correspondant à la température de $4,2{ }^{\circ} \mathrm{K}$.

Pour le métallurgiste à la recherche d'un bon matériau supraconducteur, les caractéristiques supraconductrices fondamentales sont donc:

- la température critique (ou de transition), $T_{\mathrm{c}}$, qui devra être supérieure à $4,2{ }^{\circ} \mathrm{K}$;

- le champ critique $H_{\mathrm{u}}$ à $4,2^{\circ} \mathrm{K}$ et

- la courbe $J_{\mathrm{c}}(H)$ à $4,2{ }^{\circ} \mathrm{K}$, qui doivent être les plus élevés possible.

Nous allons voir de quoi dépendent ces différentes caractéristiques.

I. TEMPÉRATURE ET CHAMP CRITIQUes. - La théoi ie et l'expérience montrent que, pour des matériaux 
possédant une résistivité résiduelle à l'état normal suffisamment élevée (ce qui est le cas des matériaux à base de $\mathrm{Nb}$ ), $T_{\mathrm{c}}$ et $H_{\mathrm{u}}$ ne dépendent pas (ou très peu) de la microstructure du matériau. Ainsi $T_{\mathrm{c}}$ et $H_{\mathrm{u}}$ peuvent être considérés comme caractéristiques d'une phase donnée à une concentration donnée.

La plus forte température critique connue actuellement, $20,7^{\circ} \mathrm{K}$, est celle d'un alliage ternaire $\mathrm{Nb}-\mathrm{Al}-\mathrm{Ge}$ et les plus forts champs critiques à $4,2{ }^{\circ} \mathrm{K}$ avoisinent 250 kOe.

Pour un alliage monophasé, il n'y a pas de relation simple entre sa température critique, celles des métaux constitutifs et leurs concentrations respectives dans l'alliage. Il en est de même pour les champs critiques qui passent généralement par un maximum pour une concentration voisine de 50 at $\%$.

Pour un alliage polyphasé, $T_{\mathrm{c}}$ et $H_{\mathrm{u}}$ sont ceux de la phase constitutive possédant la température ou le champ critique les plus élevés, car une fois supraconductrice cette phase shunte les autres.

Remarque. - Ce qui vient d'être dit à propos de la température et du champ critiques, n'est valable que pour des matériaux massifs à l'exclusion de films minces ou de très petites particules dont une dimension au moins serait de quelques dizaines d'A seulement. Dans ces cas-là interviennent des effets de taille ou des effets de proximité de la part des matériaux voisins, effets qui peuvent modifier fortement les températures et champs critiques.

II. Densité de courant CRITIQue. - Si, comme nous l'avons vu plus haut, $T_{\mathrm{c}}$ et $H_{\mathrm{u}}$ sont des propriétés d'équilibre qui dépendent très peu de la microstructure réelle d'un matériau, la densité de courant critique au contraire en dépend énormément, au point qu'il est possible de la faire varier d'un facteur $10^{3}$ à $10^{4}$ sur un même matériau en modifiant la microstructure par des traitements thermiques et mécaniques.

Cette extrême sensibilité de $J_{\mathrm{c}}$ aux variations structurales du matériau est due aux interférences entre la structure physique et la structure électromagnétique très particulière des supraconducteurs de $2^{\mathrm{e}}$ espèce que nous allons rappeler succinctement ici.

Structure électromagnétique d'un supraconducteur de $2^{e}$ espèce. - Quand un supraconducteur de deuxième espèce est soumis à un champ magnétique supérieur à quelques centaines d'Oe et inférieur à $H_{\mathrm{u}}$, le flux magnétique pénètre dans le matériau sous forme de filaments parallèles au champ extérieur.

L'âme de chaque filament est constituée d'une région où le métal est normal et porte un quantum de flux $\varnothing_{0}\left(2 \times 10^{-7}\right.$ G. $\left.\mathrm{cm}^{2}\right)$. Autour de cette âme normale tournent des courants supraconducteurs qui écrantent le champ régnant dans la région normale, vis-à-vis du reste du matériau demeuré supraconducteur. C'est pourquoi ces filaments de flux (ou lignes de flux, ou fluxoïdes) sont aussi dénommés vortex ou lignes de vortex. En fait, il n'y a pas de transition franche entre l'âme normale du vortex et la matrice supraconductrice qui l'entoure.

Tout au plus peut-on dire que la concentration en électrons supraconducteurs décroît rapidement sur une distance $\xi$, dite longueur de cohérence, depuis la matrice jusqu'à l'axe du vortex où elle est nulle ; inversement, le champ magnétique est progressivement écranté par les courants supraconducteurs sur une distance $\lambda$ dite profondeur de pénétration, depuis l'axe du vortex où il est maximum, jusqu'à la matrice supraconductrice où il est nul (Fig. 1).

Les longueurs $\lambda$ et $\xi$ sont caractéristiques d'un matériau donné à une température donnée et ne dépendent pas du champ magnétique extérieur auquel est soumis le matériau. Par contre, la densité de vortex dans le matériau dépend du champ magnétique extérieur et croît avec lui jusqu'à ce que les âmes normales des vortex se touchent et coalescent: le matériau devient alors normal et cette situation correspond à la valeur critique $H_{\mathrm{u}}$ du champ extérieur.
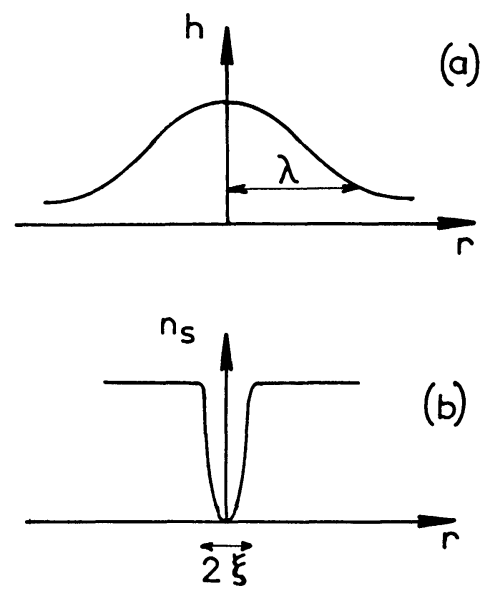

FIG. 1. - Répartition du champ magnétique $(a)$ et de la densité d'électrons supraconducteurs $(b)$ au voisinage de l'axe d'une ligne de vortex.

Ainsi, plus l'âme normale des vortex (mesurée par $\xi$ ) est petite, plus la densité de vortex nécessaire pour qu'elles se touchent est grande et plus le champ critique est élevé. Pour un matériau possédant un champ critique supérieur à $30 \mathrm{kOe}, \xi_{0}$ est inférieure à $100 \AA$ alors que $\lambda$ vaut quelques milliers d'Å. En champs magnétiques élevés, les densités de vortex sont très fortes $\left(10^{11}\right.$ vortex. $\mathrm{cm}^{-2}$ dans $20 \mathrm{kOe}, 10^{12}$ vortex. $\mathrm{cm}^{-2}$ dans $\left.200 \mathrm{kOe}\right)$.

Interactions vortex-microstructure. - Lorsqu'on fait passer un courant électrique dans le supraconducteur, les vortex sont soumis à une force électromagnétique provenant de l'action du courant sur le champ régnant dans l'âme normale du vortex. Si le matériau est parfaitement homogène et sans défauts, les vortex se déplacent sous l'action de cette force. $\mathrm{Au}$ cours de ce déplacement, de la chaleur est dégagée provenant d'un certain frottement des vortex sur le réseau cristallin, dû aux électrons normaux. Si cette 
chaleur, fonction du courant qui traverse le supraconducteur, ne peut être évacuée, la température du matériau s'élève jusqu'à $T_{\mathrm{c}}$ et le matériau devient normal. Les courants nécessaires à ce retour à l'état normal dépendent des propriétés thermiques $\mathrm{du}$ matériau et des conditions de refroidissement de l'échantillon; ils sont toujours faibles.

Si par contre, le matériau contient des inhomogénéités ou des défauts structuraux divers (ce qui est toujours le cas pour un matériau réel), ceux-ci exercent une force sur les vortex qui s'oppose à leur déplacement. Tant que cette force, dite force d'ancrage, est supérieure à la force électromagnétique exercée par le courant sur les vortex (force de Lorentz), ces derniers, ancrés sur les défauts, restent immobiles et aucun dégagement de chaleur n'a lieu dans le matériau qui reste supraconducteur.

Si on fait croître le courant qui traverse le matériau, la force de Lorentz, $F_{\mathrm{L}}$, croît jusqu'au moment où elle devient supérieure à la force d'ancrage $F_{\mathrm{a}}$; les vortex se décrochent alors brusquement des défauts, le dégagement de chaleur est important et le retour à l'état normal pratiquement instantané. La densité de courant pour laquelle les vortex se décrochent est la densité de courant critique, $J_{c}$, et l'on a alors en champ magnétique $H$ suffisamment élevé :

$$
F_{\mathrm{L}}=F_{\mathrm{a}} \# J_{\mathrm{c}} H
$$

$F_{\mathrm{a}}$, force globale par unité de volume, est la résultante de toutes les forces d'ancrage élémentaires entre vortex et centres d'ancrage. Elle va donc dépendre, non seulement de la nature des centres d'ancrage, mais aussi de leur nombre et de leur distribution, ainsi que du nombre de vortex dans le matériau donc du champ magnétique appliqué.

Les mécanismes élémentaires de l'ancrage des vortex ne sont pas connus et aucune théorie ne permet à l'heure actuelle d'expliquer quantitativement, les variations de $F_{\mathrm{a}}$ (et donc de $J_{\mathrm{c}}$ ) avec le champ magnétique appliqué, pour un matériau donné contenant un type de centre d'ancrage donné ; seules, des approches qualitatives ont été faites.

Expérimentalement on constate que $J_{c}$ décroît toujours lorsque $H$ croît, pour s'annuler au champ critique $H_{\mathrm{u}}$. On observe dans certains cas un palier de $J_{c}$ dans les champs intermédiaires et un pic plus ou moins prononcé au voisinage de $H_{\mathrm{u}}$. La figure 2 montre quelques courbes $J_{c}(H)$ caractéristiques. De même que le champ critique, $J_{\mathrm{c}}$ et $F_{\mathrm{a}}$ décroissent quand la température augmente pour s'annuler à $T_{\mathrm{c}}$, ce qui montre l'intérêt d'utiliser le supraconducteur à une température bien inférieure à sa température critique.

Centres d'ancrage. - Toute région discrète dans le matériau où les propriétés supraconductrices varient localement, en particulier $\lambda$ et $\xi$, peut constituer un centre d'ancrage.

En effet, l'énergie du vortex dépendant de $\lambda$ et $\xi$, tout gradient dans le matériau de ces deux paramètres (ou de l'un des deux) va constituer pour le vortex un

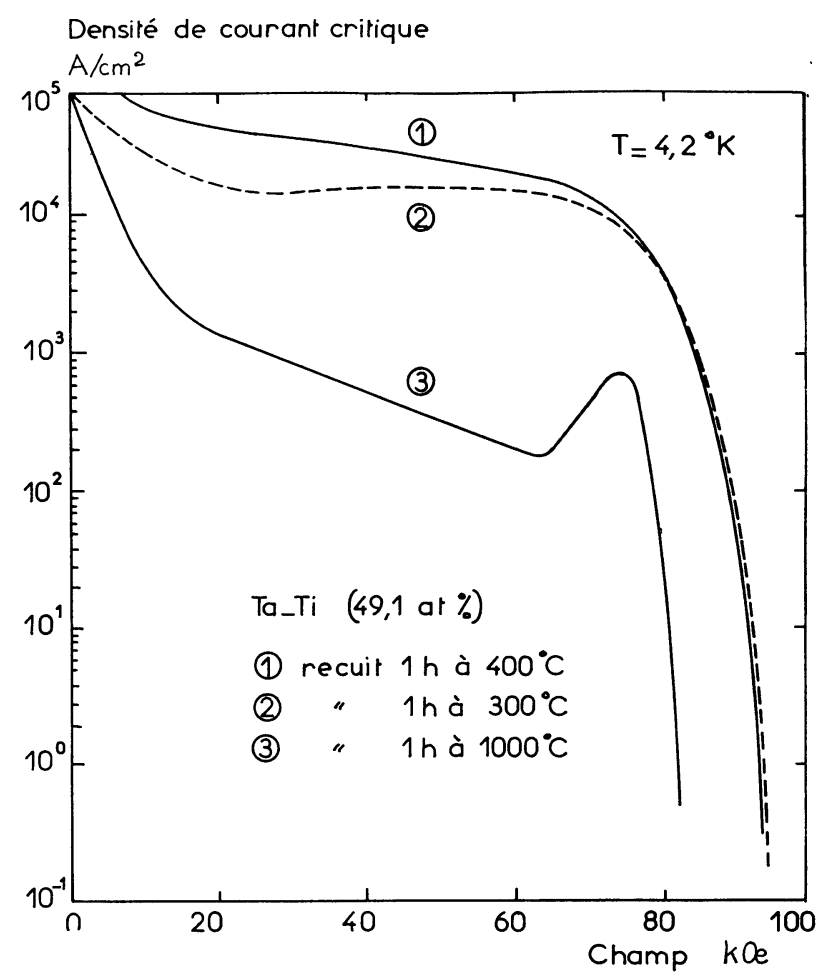

Fig. 2. - Courbes caractéristiques de densité de courant critique en fonction du champ magnétique appliqué ; (1) décroissance continue ; (2) palier en champs intermédiaires ; (3) effet de pic au voisinage du champ critique.

gradient de potentiel d'où une force résultante qui sera attractive ou répulsive suivant le signe du gradient. Il en résulte que les dimensions des centres d'ancrage doivent être au moins de l'ordre de grandeur de $\xi$, le plus petit des 2 paramètres, ce qui exclut les défauts ponctuels (lacunes, interstitiels) et les très petits défauts tels que les groupes de quelques atomes (clusters). On a par exemple montré que des zones de Guinier-Preston de quelques dizaines d' $\AA$ de diamètre n'ancraient pas les vortex dans des alliages d'Al où $\lambda$ et $\xi$ ont des valeurs de l'ordre du millier d'À.

Les centres d'ancrage vont donc être constitués par tous les défauts suffisamment étendus du réseau cristallin :

- fluctuations locales de concentration, ségrégations, précipités,

- dislocations, joints de grains, parois de cellules de polygonization,

- défauts d'irradiation, etc...

En raison des difficultés de préparation d'échantillons contenant des défauts bien contrôlés, très rares sont les cas où, pour des centres d'ancrage donnés, on connaît les paramètres qui gouvernent la force d'ancrage: taille des centres, forme, nature, concentration, répartition. Néanmoins, certaines expériences semblent montrer que la force d'ancrage est proportionnelle à la surface totale des centres par unité de volume dans le cas de gros précipités 
(alliages eutectiques), de joints de grains ou de cellules de polygonization.

Par ailleurs, il semble d'une manière générale, que des précipités d'une phase normale ancrent davantage les vortex que des précipités d'une phase supraconductrice. Pour les dislocations, l'ancrage semble plus fort lorsqu'elles sont regroupées en amas que lorsqu'elles sont uniformément distribuées.

$\mathrm{Si}$, comme on vient de voir, on connaît fort mal les détails des mécanismes d'ancrage des vortex, on sait par contre quels sont les traitements métallurgiques qui permettent de faire varier $J_{c}$ dans de grandes proportions; ce sont les traitements mécaniques d'écrouissage, les traitements thermiques et pour les alliages de $\mathrm{Nb}$ en particulier, l'addition d'impuretés interstitielles.

Deuxième partie. - LES MATÉRIAUX SUPRACONDUCTEURS A BASE DE NIOBIUM. - Ils peuvent être classés en deux catégories distinctes.

- Les alliages ductiles qui n'ont pas les plus hautes performances supraconductrices mais qui peuvent aisément être transformés en fils fins nécessaires à la confection des bobinages;

- Les alliages ordonnés qui possèdent les plus hautes performances supraconductrices connues mais qui sont fragiles et dont la fabrication sous forme de fils ou rubans demande des techniques particulières.

I. Les Alliages DuCTILES. - Ce sont essentiellement les alliages du $\mathrm{Nb}$ avec les métaux du groupe IV B du tableau périodique, Ti, $\mathrm{Zr}$, Hf.

Chronologiquement les alliages $\mathrm{Nb}-\mathrm{Zr}$ ont été les premiers matériaux supraconducteurs commercialisés. Ils ont été supplantés par des alliages $\mathrm{Nb}$-Ti aux performances plus élevées et aux prix de revient inférieurs. L'intérêt des alliages $\mathrm{Nb}$-Hf n'est pas commercial mais réside dans le fait que possédant des champs critiques moins élevés que ses congénères ils se prêtent plus aisément aux mesures.

Bien que leurs diagrammes d'équilibre soient parfois différents (Fig. 3), ces systèmes binaires ont ceci de commun que la solution solide cubique centrée haute température $\beta$, est généralement retenue à la température ambiante pour les alliages contenant plus de 30 at \% environ de $\mathrm{Nb}$. Pour les concentrations de plus en plus faibles en $\mathrm{Nb}$, apparaissent successivement sur les alliages refroidis rapidement ou trempés, une phase métastable $\omega$ hexagonale correspondant à une légère déformation de la phase $\beta$, une phase $\alpha^{\prime \prime}$ tétragonale puis la phase martensitique $\alpha^{\prime}$ hexagonale compacte (phase $\alpha$ sursaturée). Notons que la phase $\omega$ est susceptible de se former et d'évoluer en concentration au cours de traitements thermiques dans le domaine $(\alpha+\beta)$ avant que la phase $\alpha$ ne précipite et que les domaines d'existence de ces phases métastables ainsi que leurs conditions d'apparition sont très mal connus à l'heure actuelle.

En conséquence nous examinerons séparément le

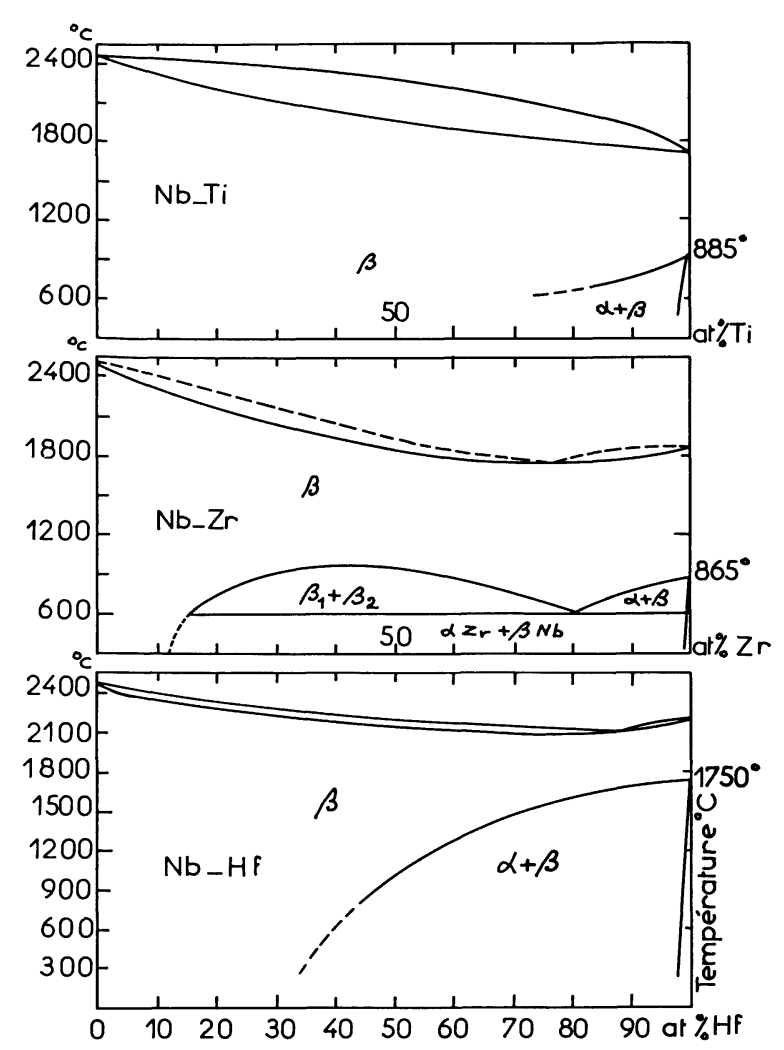

FIG. 3. - Diagrammes de phases des systèmes binaires $\mathrm{Nb}-\mathrm{Ti}$, $\mathrm{Nb}-\mathrm{Zr}, \mathrm{Nb}-\mathrm{Hf}$.

comportement supraconducteur des alliages dans les domaines monophasé et bi- ou polyphasé.

Domaine monophasé. - Sur la figure 4 ont été Tempéralure

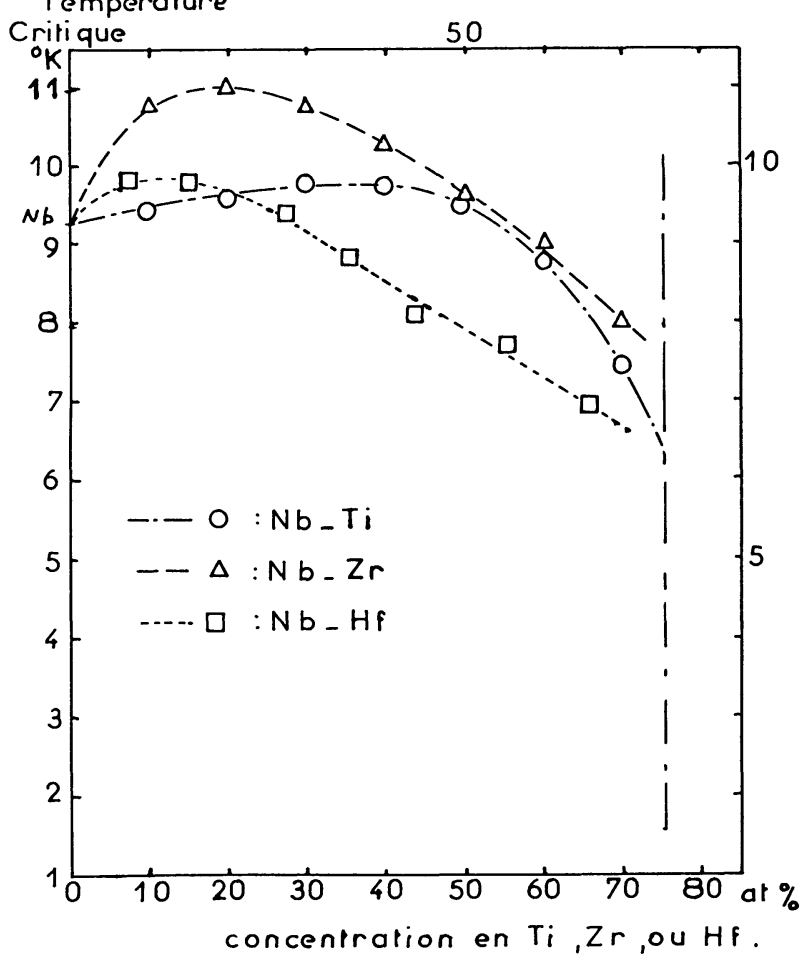

FIG. 4. - Températures critiques en fonction de la concentration dans le domaine monophasé $\beta$ des systèmes binaires $\mathrm{Nb}-\mathrm{Ti}$, $\mathrm{Nb}-\mathrm{Zr}, \mathrm{Nb}-\mathrm{Hf}$. 
reportées les températures critiques des 3 systèmes binaires. On peut voir que dans tout le domaine $\beta$, les $T_{\mathrm{c}}$ sont nettement supérieures à $4,2^{\circ} \mathrm{K}$.

Sur la figure 5 ont été reportés les champs critiques mesurés par apparition de la résistance sur des fils pour les alliages $\mathrm{Nb}-\mathrm{Ti}$ et $\mathrm{Nb}-\mathrm{Zr}$ et par disparition de l'aimantation supraconductrice pour les alliages $\mathrm{Nb}-\mathrm{Hf}$.

D’une manière générale la densité de courant critique dans les champs intermédiaires décroît lorsque la concentration en soluté dans le $\mathrm{Nb}$ augmente comme on peut le voir sur la figure 6 où ont été reportées pour diverses concentrations en $\mathrm{Hf}$ des courbes $J_{\mathrm{c}}(H)$ obtenues sur des échantillons qui ont tous subi le même traitement métallurgique (forgeage à chaud, martelage à froid).

L'effet de l'écrouissage sur $J_{\mathrm{c}}$ est considérable et on observe couramment une réduction de $J_{\mathrm{c}}$ d'un facteur 1000 entre un échantillon fortement écroui

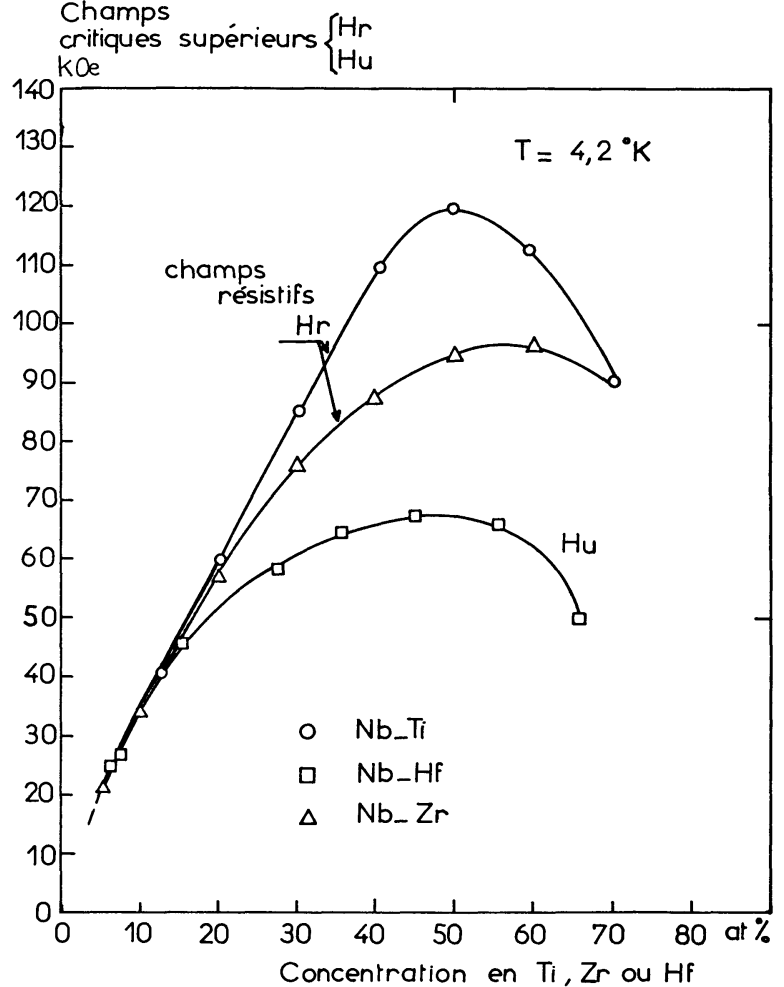

FIG. 5. - Champs critiques à $4,2^{\circ} \mathrm{K}$ en fonction de la concentration dans le domaine monophasé $\beta$ des systèmes binaires $\mathrm{Nb}-\mathrm{Ti}, \mathrm{Nb}-\mathrm{Zr}, \mathrm{Nb}-\mathrm{Hf}\left({ }^{*}\right)$. Les déterminations du champ critique ont été faites soit par mesure de la résistance $\left(H_{\mathrm{r}}\right)$ soit par mesure d'aimantation $\left(H_{\mathrm{u}}\right)$

et le même échantillon bien recuit. L'influence de l'écrouissage sefait encore sentir pour des taux élevés comme le montre la figure 7. L'effet de l'écrouissage est attribué aux dislocations qui peuvent agir sur les vortex soit par elles-mêmes, soit en constituant des régions de ségrégation ou précipitation préférentielle

(*) J. Y. Guérin, thèse, Grenoble 1970 (à paraître). ReVUe de PHYSiQue APPLIQUÉE. - T. 5, No 3, JUIN 1970

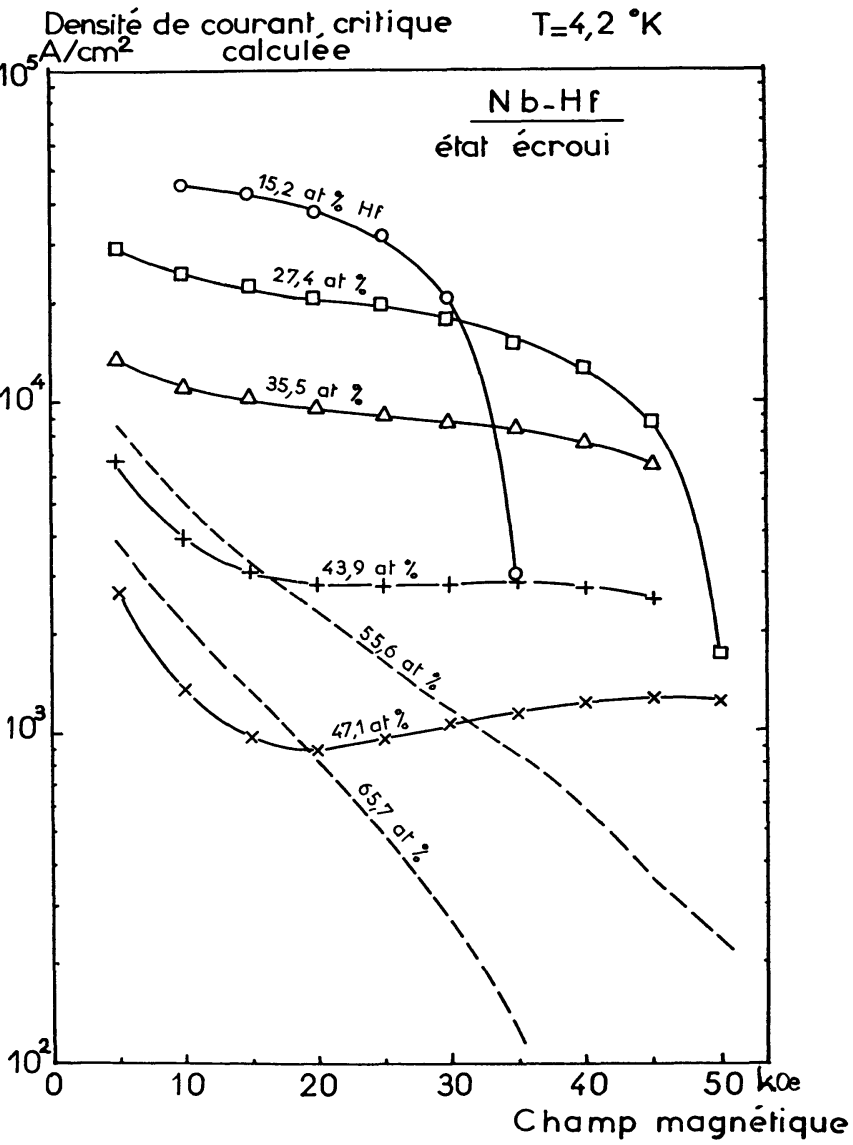

Fig. $6\left(^{*}\right)$. - Densité de courant critique calculée à partir des courbes d'aimantation, en fonction du champ magnétique appliqué pour des alliages $\mathrm{Nb}$-Hf de diverses concentrations. Les alliages ont été forgés à chaud en carrés de $9 \mathrm{~mm}$ de côté puis martelés à froid jusqu'à un diamètre de $3 \mathrm{~mm}$.

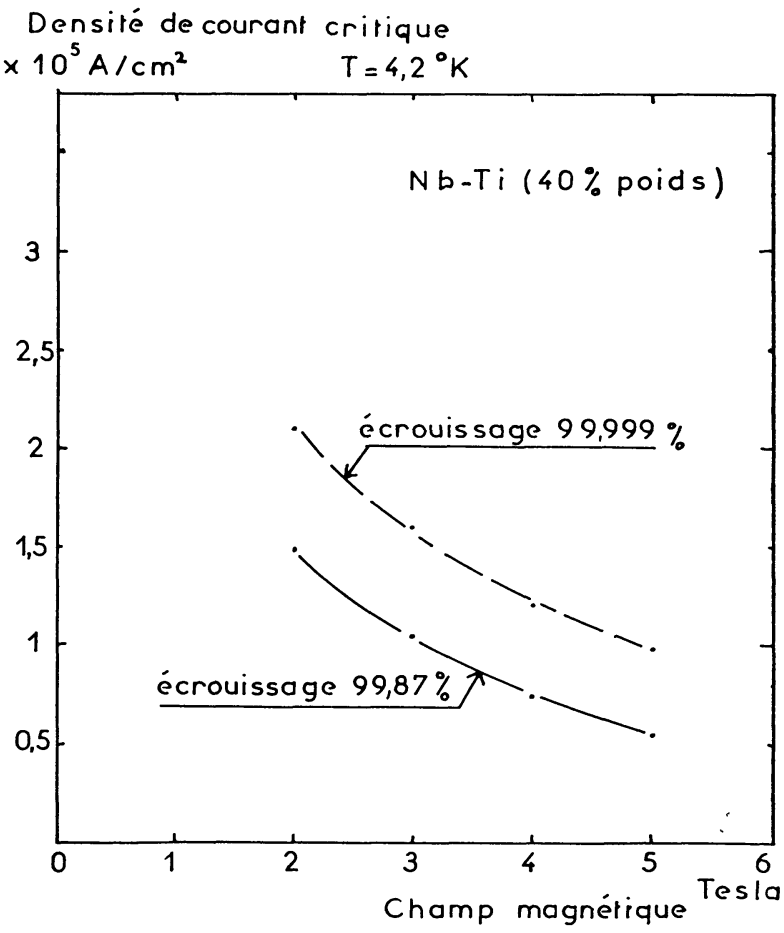

Fig. 7. - Densité de courant critique mesurée en fonction du champ magnétique appliqué, pour deux fils d'alliage $\mathrm{Nb}$ - $\mathrm{Ti}$ $40 \%$ ayant subi des taux d'écrouissage différents. 
pour les impuretés interstitielles. Quoique certains auteurs soutiennent qu'une distribution homogène de dislocations puisse ancrer efficacement les vortex, il semble bien que ce soit les empilements de dislocations suffisamment étendus qui jouent le rôle de centres d'ancrage dans ces alliages. En effet, dans les alliages de $\mathrm{Nb}$ fortement écrouis, les dislocations se regroupent dans les parois de cellules qui s'allongent dans le sens du laminage ou du tréfilage au point de donner une structure fibreuse. A l'observation au microscope électronique on distingue des sous-bandes ou micro bandes orientées parallèlement à la direction de tréfilage. On a pu constater que $J_{\mathrm{c}}$ variait dans le même sens que la densité de ces microbandes qui peut atteindre $10^{12} \mathrm{~cm}^{-2}$ soit un espacement de $100 \AA$ sur des fils fins. Par ailleurs on a constaté un fort effet d'anisotropie, $J_{\mathrm{c}}$ étant maximum lorsque les vortex sont parallèles aux microbandes. Par ailleurs, il ne semble pas que les parois des cellules de dislocations et les joints de grains aient un effet différent sur $J_{c}$; en effet, sur un même matériau ( $\left.\mathrm{Nb}-\mathrm{Zr} 33 \%\right)$ différemment écroui ou recristallisé, $J_{c}$ suit une loi monotone en $1 / d, d$ représentant aussi bien le diamètre moyen des cellules de dislocation que celui des joints de grains.

Généralement, $J_{\mathrm{c}}$ passe par un maximum en fonction de la température de traitement thermique effectué après écrouissage, comme on peut le voir sur la figure 8. L'accroissement de $J_{\mathrm{c}}$ jusqu'au maximum, serait dû au renforcement de la structure cellulaire des dislocations qui quittent le centre des cellules pour s'accumuler dans les parois. La décroissance ultérieure de $J_{\mathrm{c}}$ pourrait être due au départ de dislocation et au grossissement des cellules.

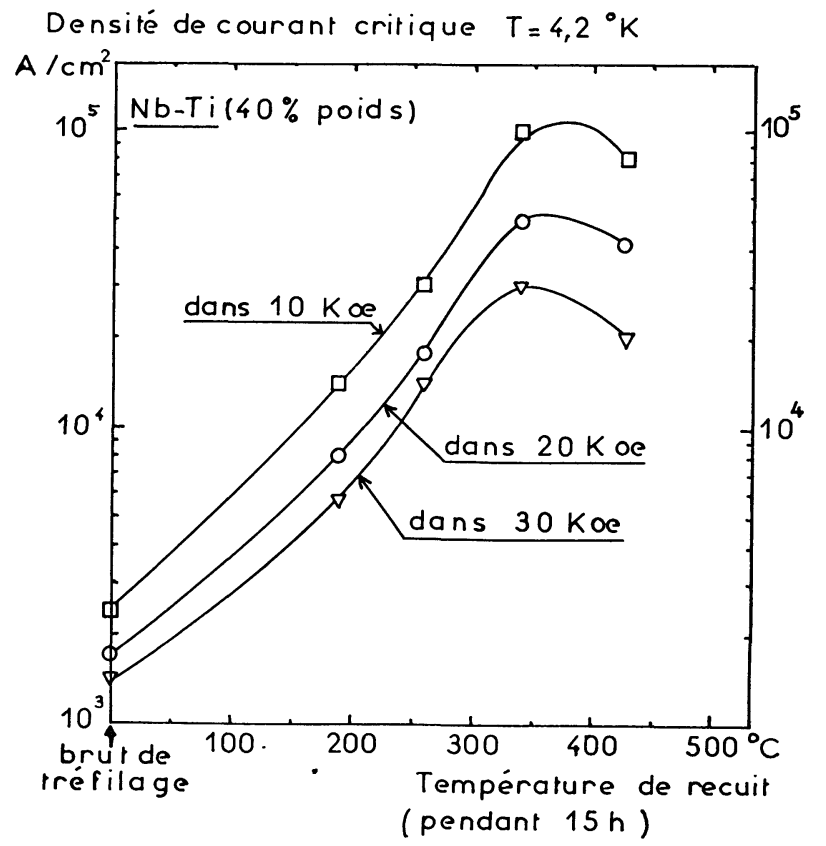

Fig. 8. - Densité de courant critique mesurée sur des fils d'alliage $\mathrm{Nb}-\mathrm{Ti} 40 \%$, dans des champs magnétiques de 10,20 et $30 \mathrm{kOe}$, en fonction de la température de traitement thermique.
L'introduction d'impuretés interstitielles, azote et surtout oxygène, augmente $J_{c}$, même lorsqu'il n'apparaît aucune précipitation de ces impuretés. Leur rôle n'est à l'heure actuelle pas très clair et il semble qu'elles renforcent l'effet des dislocations en se ségrégant sur elles. Par ailleurs, même à des teneurs très faibles (de l'ordre de $100 \mathrm{ppm}$ ), l'oxygène accélère fortement la précipitation de la phase $\alpha$.

Domaine biphasé ou polyphasé. - Le comportement supraconducteur des alliages dans ce domaine est très complexe, comme la structure elle-même qui n'est généralement pas assez bien connue pour permettre une interprétation non ambiguë des propriétés supraconductrices.

D’une manière générale, la précipitation de phase $\alpha$, à condition d'être très fine, augmente $J_{c}$, un facteur 10 étant couramment atteint. La finesse de la précipitation est obtenue par traitement thermique après écrouissage à des températures modérées, de l'ordre de $400{ }^{\circ} \mathrm{C}$ pour les alliages $\mathrm{Nb}$-Ti. Dans ce cas les précipités ont des dimensions variant de 300 à $500 \AA$ et constituent des centres d'ancrage très efficaces. Ils sont localisés préférentiellement aux limites des microbandes, la structure fibreuse d'écrouissage étant conservée.

$\mathrm{Si}$, au contraire, la précipitation est effectuée à température élevée, les précipités sont grossiers, la structure d'écrouissage est détruite et la densité de courant est abaissée.

Les particules de phase $\omega$, en raison de leur petite taille constituent aussi des centres d'ancrage très efficaces si leur concentration est différente de celle de la phase $\beta$, ce qui est obtenu ici aussi, par traitement thermique à température modérée $\left(400-450^{\circ} \mathrm{C}\right.$ pour un alliage $\mathrm{Ti}-\mathrm{Nb} 22$ at \% préalablement trempé). Néanmoins, la formation de phase $\omega$ entraînant un accroissement important de la dureté de l'alliage préjudiciable à sa transformation en fil, on cherche plutôt à l'éviter en choisissant correctement les traitements thermiques.

Signalons enfin qu'une alternance de traitements thermiques et mécaniques donne des $J_{\mathrm{c}}$ plus élevées qu'un simple traitement thermique final.

En conclusion, nous pouvons dire qu'en raison du caractère très qualitatif de nos connaissances de la microstructure des alliages et de ses interactions avec les vortex, l'optimisation des performances d'un alliage supraconducteur reste encore dans le domaine semi-empirique et nécessite de très nombreux essais.

Les alliages $\mathrm{Nb}$ - $\mathrm{Ti}$ actuellement commercialisés contiennent de 40 à $60 \%$ en poids de Ti. Leurs densités de courant critique sont de l'ordre de $10^{5} \mathrm{~A} . \mathrm{cm}^{-2}$ dans des champs magnétiques atteignant $80 \mathrm{kOe}$ (ce qui représente un courant de $40 \mathrm{~A}$ dans un fil de $0,25 \mathrm{~mm}$ de diamètre). Deux nuances sont produites en France : 40 et $57 \%$ en poids de Ti. Les alliages sont élaborés par Ugine-Kuhlmann puis transformés, traités thermiquement et stabilisés par Thomson-CSF qui 
les vend sous forme de fils monobrins ou de câbles multibrins.

II. LeS ALliages ORDONNÉS. - C'est parmi les composés intermétalliques de structure A 15 (type $\beta \mathrm{W}$ ) et de formule $\mathrm{A}_{3} \mathrm{~B}$, qu'on trouve les supraconducteurs possédant les températures et champs critiques les plus élevés.

$\begin{array}{ccccc}\text { Com- } & \mathrm{Nb}_{3} & \mathrm{Nb}_{3} \mathrm{Sn} & \mathrm{Nb}_{3} \mathrm{Al} & \mathrm{V}_{3} \mathrm{Si} \\ \text { posé } & \left(\mathrm{Al}_{0,8} \mathrm{Ge}_{0,2}\right) & -\overline{18} & \overline{17,5} & \overline{17} \\ \overline{T_{\mathrm{c}}{ }^{\mathrm{o}} \mathrm{K}} & 20,7 & 18 & \end{array}$

$\mathrm{Nb}_{3}\left(\mathrm{Al}_{0,8} \mathrm{Ge}_{0_{2}}\right)$ a été découvert récemment (1967) et peu d'études ont encore été effectuées à son sujet. Par contre $\mathrm{Nb}_{3} \mathrm{Sn}$ a été pendant une dizaine d'années le matériau supraconducteur possédant la plus haute température critique et, malgré ses qualités mécaniques déplorables (allongement maximum à la rupture de 0,2 à $0,4 \%$ ), ses performances supraconductrices ont suscité son étude et son développement industriel. C'est à l'heure actuelle le seul alliage ordonné supraconducteur à être produit industriellement. Qualitativement les divers composés $A_{3} B$ se comportent de la même manière et nous nous limiterons dans ce qui suit au seul composé $\mathrm{Nb}_{3} \mathrm{Sn}$.

Structure et propriétés supraconductrices. - La structure des composés $\mathrm{A}_{3} \mathrm{~B}$ et le diagramme d'équilibre $\mathrm{Nb}-\mathrm{Sn}$ sont représentés sur la figure 9 . On peut voir que les atomes de $\mathrm{Nb}$ forment des chaînes linéaires orthogonales qui s'évitent les unes les autres. C'est cette structure linéaire des atomes de $\mathrm{Nb}$ qui est res-
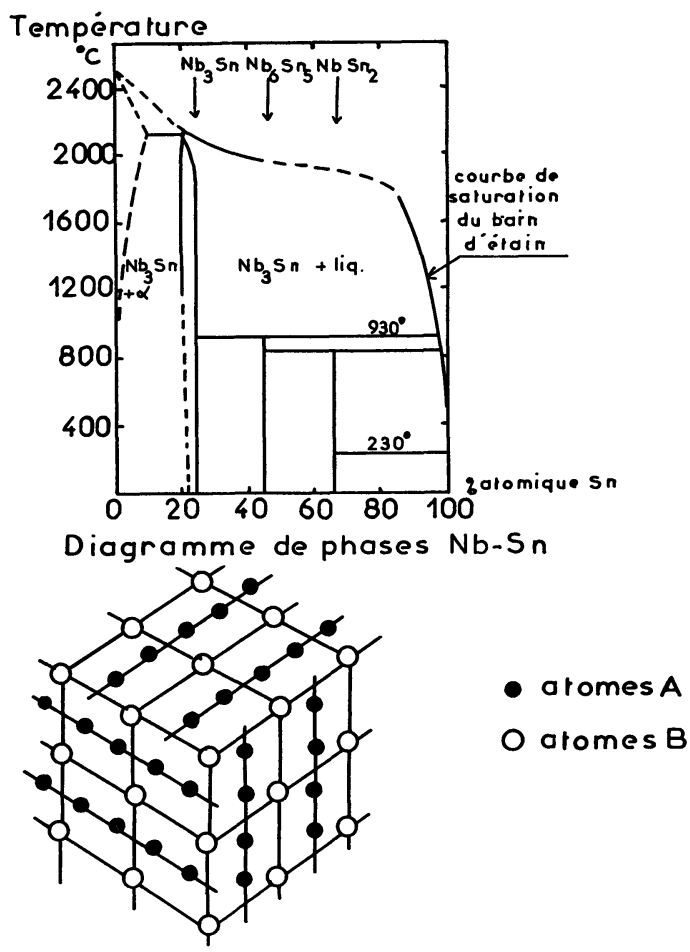

STRUCTURE A-15 $A_{3} B$

Fig. 9. - Diagramme de phases du système binaire Nb-Sn et structure cristalline des alliages ordonnés $\mathrm{A}_{15}$. ponsable de la température critique élevée par l'intermédiaire d'une structure de bande particulière pour les électrons $d$, présentant des pics de densité d'état.

La préparation difficile de $\mathrm{Nb}_{3} \mathrm{Sn}$, qui se fait généralement par frittage et diffusion en raison de la volatilité de $\mathrm{Sn}$, et ses mauvaises propriétés mécaniques qui interdisent toute transformation plastique, ont limité considérablement les études de l'influence de la structure sur les caractéristiques supraconductrices en particulier sur la densité de courant critique qui ne peut être mesurée que sur des échantillons de très petite section.

La caractéristique supraconductrice la plus étudiée car ne nécessitant pas de formes particulières pour les échantillons, a été la température critique. D'une manière générale $T_{\mathrm{c}}$ est maximale pour la composition stœchiométrique et le degré d'ordre maximal de la structure. La figure 10, où a été portée $T_{\mathrm{c}}$ en fonction de la concentration en $\mathrm{Sn}$ pour des échantillons fondus, avant et après recuit illustre bien ce fait.

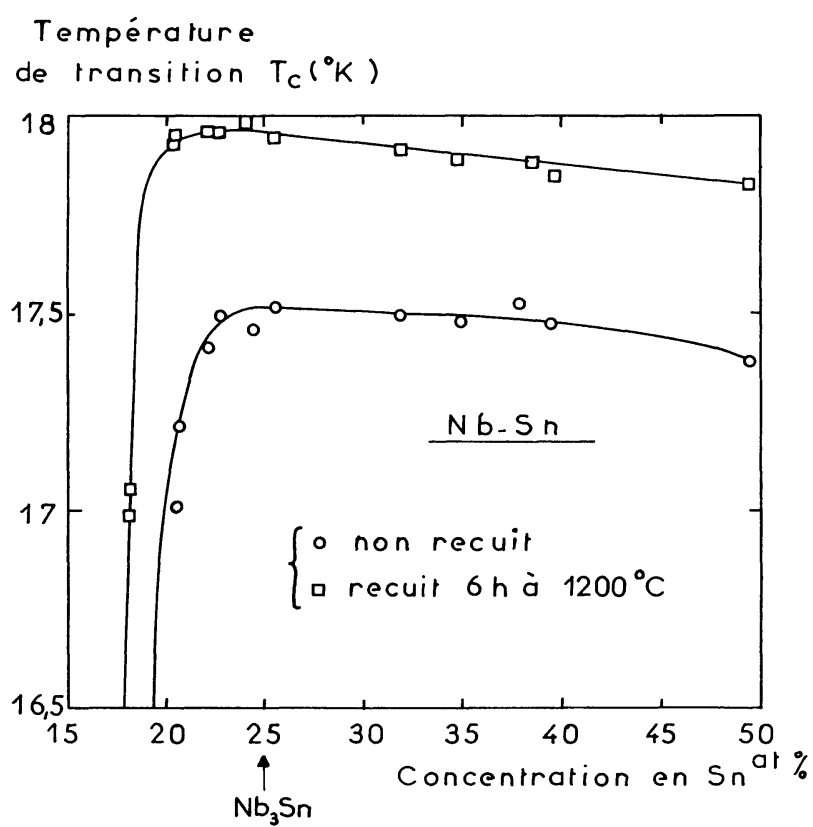

Fig. 10. - Température critique en fonction de la concentration en $\mathrm{Sn}$ pour le système Nb-Sn. Les échantillons ont été fondus à l'arc sous pression réduite d'argon.

La plupart des mesures de champ critique ont été effectuées à des températures voisines de $T_{\mathrm{c}}$ car le champ critique à $4,2{ }^{\circ} \mathrm{K}$ est trop élevé pour être mesuré commodément. Néanmoins quelques mesures effectuées à $4,2^{\circ} \mathrm{K}$ en champ pulsé, indiquent un champ critique de $225 \mathrm{kOe}$. L'influence de la structure sur le champ critique n'a pas été étudiée jusqu'ici.

En ce qui concerne la densité de courant critique, toutes les mesures ont été faites sur des couches minces où il n'est guère possible de faire varier fortement la micro-structure. Les variations de $J_{c}$ observées lorsque l'on modifie un paramètre du mode de fabrication, n'ont de sens, en général, que par rapport à ce mode de fabrication et l'influence particulière sur $J_{\mathrm{c}}$ de tel ou tel défaut structural n'a jamais été 
étudiée sérieusement. En conséquence, il n'est pas possible de donner ici, comme pour les alliages ductiles, un aperçu des facteurs métallurgiques qui gouvernent la densité de courant. Tout au plus peut-on dire qu'en général, un bon recuit abaisse toujours $J_{c}$, en éliminant les défauts structuraux présents.

Procédés de fabrication des conducteurs souples. Pour les applications il est nécessaite de produire des conducteurs propres à la réalisation de bobinages. Les procédés utilisés ou envisagés peuvent être classés en deux groupes :

- les procédés Kunzler et variantes, qui consistent à tréfiler un tube de $\mathrm{Nb}$ contenant un mélange de poudres de $\mathrm{Nb}$ et de $\mathrm{Sn}$, à le bobiner sur un mandrin réfractaire et à traiter le tout à $950^{\circ} \mathrm{C}$ pour faire former $\mathrm{Nb}_{3} \mathrm{Sn}$ par diffusion. Ce procédé, qui a permis de réaliser le premier gros bobinage supraconducteur aux Etats-Unis et qui a été étudié en France par la Société SODERN, a été abandonné en raison de son manque de souplesse, le conducteur n'étant pas récupérable après cuisson du bobinage.

- Les procédés qui consistent à fabriquer une mince couche de $\mathrm{Nb}_{3} \mathrm{Sn}$ sur un substrat qui pourra être un ruban suffisamment souple pour être bobiné. Une souplesse suffisante de la couche de $\mathrm{Nb}_{3} \mathrm{Sn}$ est obtenue ici, d'une part grâce à sa faible épaisseur (quelques microns) et d'autre part, grâce au fait que, pour un ruban, la couche est plus proche de la fibre neutre que pous un fil, et subit donc des efforts de traction moindres lors d'une courbure.

Dans ces procédés, la couche de $\mathrm{Nb}_{3} \mathrm{Sn}$ est obtenue soit :

- par co-déposition de $\mathrm{Nb}$ et $\mathrm{Sn}$ sur un ruban qui peut être quelconque (procédé RCA par décomposition des chlorures, dépôt par pulvérisation cathodique, projection au chalumeau à plasma, etc...) ;

- par co-laminage de $\mathrm{Nb}$ et $\mathrm{Sn}$, suivi d'un traitement de diffusion.

- par trempage d'un ruban de $\mathrm{Nb}$ dans un bain d'étain, procédé le plus couramment utilisé (General Electric, Plessey, Thomson-CSF).

Sur la figure 11 a été reproduit le schéma de principe de fabrication du ruban Thomson-CSF. L'épaisseur du ruban de $\mathrm{Nb}$ est de 10 à $20 \mu$, l'épaisseur de la couche de $\mathrm{Nb}_{3} \mathrm{Sn}$ formée est de 2 à $3 \mu$, la largeur du ruban pouvant varier entre 3 et $60 \mathrm{~mm}$. La température du bain d'étain est maintenue entre 870 et $950^{\circ} \mathrm{C}$ et la pression dans l'enceinte entre $10^{-5}$ et $10^{-6}$ torr. Avant immersion dans le bain d'étain, le ruban de $\mathrm{Nb}$ est préchauffé par effet Joule à $700^{\circ} \mathrm{C}$. Après immersion dans l'étain il est chauffé toujours par effet Joule à $900^{\circ} \mathrm{C}$ pour parfaire la diffusion de $\mathrm{Sn}$ dans le $\mathrm{Nb}$.

Avec ce procédé il a été constaté que la densité de courant était maximale lorsque l'écrouissage préalable du ruban de $\mathrm{Nb}$ était compris entre 93 et $95 \%$ ce qui laisse supposer que la micro structure initiale du ruban a un effet sur celle de la couche de $\mathrm{Nb}_{3} \mathrm{Sn}$ for-

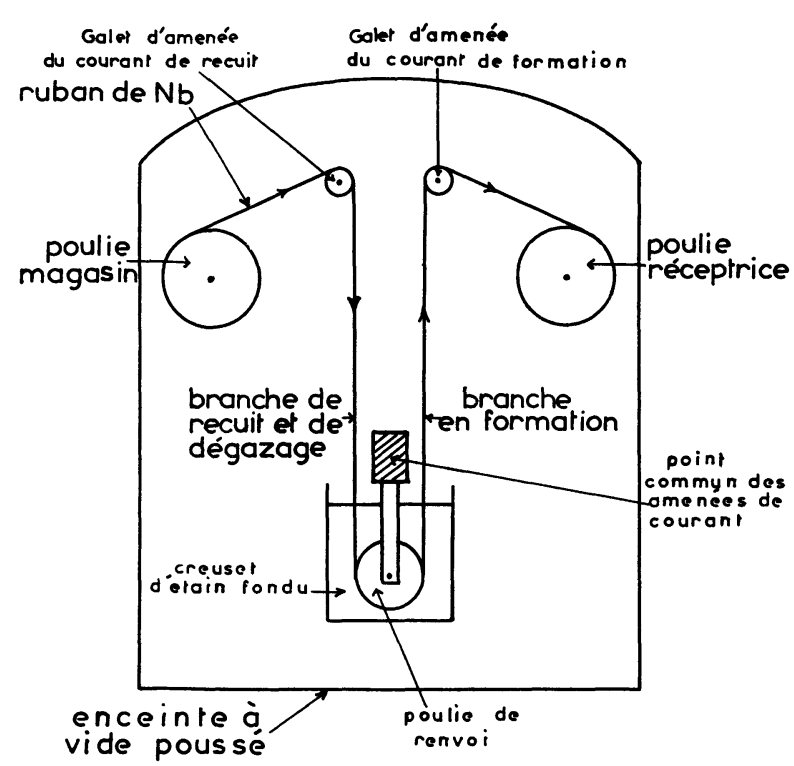

FIG. 11. - Schéma de principe de la fabrication du ruban niobium-étain Thomson-CSF (explications dans le texte).

mée. Par ailleurs, si la fabrication du ruban est effectuée sous atmosphère d'oxygène (pression de l'ordre de $10^{-3}$ torr), $J_{c}$ est abaissée, et une atmosphère réduite d'azote n'a pas d'effet appréciable sur $J_{\mathrm{c}}$.

De tels rubans possèdent des densités de courant variant entre $1,25 \times 10^{5}$ et $2,5 \times 10^{4} \mathrm{~A} . \mathrm{cm}^{-2}$ dans des champs magnétiques compris entre 40 et $130 \mathrm{kOe}$.

Troisième partie. - STABILISATION DES MATÉRIAUX SUPRACONDUCTEURS. - Instabilité des supraconducteurs à haute densité de courant critique. - Etant donné, d'une part la diversité des défauts présents dans le réseau cristallin d'un matériau et d'autre part, leur répartition, nécessail ement inhomogène à l'échelle microscopique, on conçoit que la force d'ancrage exercée par ces défauts sur les vortex, ne soit pas partout la même dans le matériau.

Dans ces conditions, certains vortex vont se décrocher prématurément des centres d'ancrage dans certaines régions du matériau; il en résulte une production de chaleur locale qui, si elle n'est pas évacuée rapidement, va élever la température localement. Nous avons vu que la force d'ancrage décroît lorsque la température s'élève, si bien que l'élévation locale de température va provoquer le décrochage de vortex voisins. On a alors un phénomène d'avalanche dit saut de flux dont la propagation va dépendre de la manière dont peut être évacuée la chaleur produite.

Ces sauts de flux, apparents sur les courbes d'aimantation, ont pu être filmés et leur propagation étudiée Si le saut de flux est important, il peut entraîner le retour prématuré du matériau à l'état normal.

Lorsqu'il s'agit d'un conducteur de grande longueur, comme c'est le cas dans les bobinages, un saut de flux peut entraîner le retour à l'état normal d'une petite longueur de fil qui va se mettre à chauffer par effet Joule et rendre normal de proche en proche tout le bobinage. Cette instabilité des matériaux supra- 
conducteurs est d'autant plus grande que $J_{\mathrm{c}}$ est plus élevée. Par ailleurs, un saut de flux peut être engendré par une cause extérieure telle que élévation locale de température, vibration, déplacement d'une spire sous l'effet des forces électromagnétiques, etc..., ce qui rend l'instabilité aléatoire. Etant donné les importantes éner gies stockées dans les bobinages supraconducteurs, tout retour intempestif à l'état normal est à éviter si l'on ne veut pas détériorer définitivement le bobinage.

Stabilisation externe. - On a cherché à remédier à cette instabilité des matériaux supraconducteurs, d'une part en réalisant des fils où le rapport surfacevolume soit le plus élevé possible de façon à améliorer le refroidissement (conducteurs multibrins où chaque brin élémentaire a un diamètre de $10 \mu$ ), d'autre part en enrobant le matériau supraconducteur avec un métal normal bon conducteur de la chaleur et du courant électrique. Le rôle de ce métal stabilisant est d'une part d'améliorer l'évacuation de la chaleur produite dans le matériau supraconducteur au cours d'un saut de flux, grâce à sa capacité calorifique et à l'augmentation de la surface de contact avec l'hélium liquide réfrigérant, d'autre part de shunter électriquement, pendant un cours instant, une petite longueur de fil supraconducteur devenue prématurément normale à la suite d'un saut de flux.

Des théories ont été faites, reposant sur le bilan thermique lors d'un saut de flux, mais elles sont souvent inapplicables en raison du manque de données thermiques à $4,2^{\circ} \mathrm{K}$. Là aussi, il faut effectuer des essais semi-empiriques pour déterminer l'épaisseur optimum du stabilisant. Les métaux utilisés comme stabilisants sont le cuivre (Thomson-CSF) et l'aluminium (C. G. E.). Le cuivre est co-tréfilé avec les alliages $\mathrm{Nb}$-Ti lors de la fabrication des conducteurs monobrins ou des multibrins cylindriques. Pour les conducteurs multibrins en bandes, dits forte intensité l'enrobage de $\mathrm{Cu}$ est réalisé par plaquage. Les conducteurs stabilisés à l'aluminium sont filés à la presse. Le ruban $\mathrm{Nb}_{3} \mathrm{Sn}$ est stabilisé au $\mathrm{Cu}$. Une première couche est déposée électrolytiquement puis une deuxième couche de $\mathrm{Cu}$ est soudée à l'étain. On peut réaliser ainsi des conducteurs contenant plusieurs rubans élémentaires de $\mathrm{Nb}_{3} \mathrm{Sn}$.

Pour les grosses installations la stabilisation a été poussée à un point tel qu'il n'y a plus de véritable transition du supraconducteur, le $\mathrm{Cu}$ étant fort capable à lui seul de supporter toute l'intensité, avec des pertes, bien entendu. A ce point, les conducteurs peuvent être considérés comme étant du $\mathrm{Cu}$ simplement fourré de matériau supraconducteur puisque celui-ci ne représente plus que $2,5 \%$ de la masse du conducteur. Si la stabilisation externe est un palliatif technologique à l'instabilité des matériaux supraconducteurs par contre une solution beaucoup plus élégante pourrait être trouvée en supprimant cette instabilité, ce qui ne semble pas impossible en principe et nécessiterait la mise au point de matériaux dans lesquels $J_{\mathrm{c}}$ augmenterait avec la température au voisinage de $4,2{ }^{\circ} \mathrm{K}$.

Conclusion. - Les matériaux supraconducteurs à base de niobium sont arrivés, à l'heure actuelle, grâce à des recherches technologiques importantes et malgré des propriétés mécaniques parfois très défavorables, à des performances et un degré de mise au point très élevés.

Néanmoins, tant qu'une description quantitative de l'ancrage des lignes de vortex ne pourra être faite, et en particulier tant que l'on n'aura pas trouvé comment varie la force d'ancrage avec la nature des centres d'ancrage, il ne sera pas possible d'assurer une limite précise aux performances des supraconducteurs en ce qui concerne le transport du courant continu. Cette description étant liée à une connaissance précise de la microstructure et à une grande maîtrise des paramètres qui la conditionnent, il semble que ce soit dans la voie de l'investigation structurale à l'échelle de la centaine d'A, ou au-dessous, que doit être poursuivi l'effort de recherche sur les matériaux supraconducteurs. Par ailleurs, du point de vue technologique, l'avenir des supraconducteurs semblant être lié à celui des forts champs magnétiques, les efforts doivent être poursuivis dans la recherche et la mise au point des matériaux à haute température critique qui corrélativement possèdent des champs critiques très élevés. Telles ont été les conclusions des tables rondes tenues lors du Colloque sur les matériaux supraconducteurs, organisé par la D. G. R. S. T. et la D. R. M. E. à Aussois (Savoie) en mars 1969.

\section{Bibliographie}

\section{Ouvrages généraux}

\section{Théorie de la supraconductivité.}

De Gennes, Superconductivity of metals and alloys, Benjamin, 1966.

SaInT-James, Thomas and SARma, Type II Superconductivity, Pergamon, 1969.
Relations structure métallurgique, propriétés supraconductrices.

LIVINGSTON and ScHADLER, The effect of metallurgical variables on superconducting properties, Progress in Materials Science, Pergamon, 1964.

SAVITSki, Baron, Efimov, BitchKova, Mitzenkova, Métallurgie des matériaux supraconducteurs, Editions de Moscou, 1969 (en russe). 